\title{
Case of early-disseminated Rhizopus microsporus var. microsporus mucormycosis in a renal transplant patient
}

This article was published in the following Dove Press journal:

International Medical Case Reports Journal

8 June 2016

Number of times this article has been viewed

\section{Dikshya Sharma' \\ Kumud Dahal ${ }^{2}$ \\ Bandana Pathak ${ }^{3}$ \\ Udip Dahal ${ }^{4}$}

'Staten Island University Hospital, Staten Island, NY, ${ }^{2}$ University of Illinois College of Medicine, ${ }^{3} \mathrm{OSF}$ Saint Francis Medical Center, Peoria, IL, ${ }^{4}$ University of Utah, Salt Lake City, UT, USA
Correspondence: Dikshya Sharma Staten Island University Hospital, 475 Seaview Avenue, Staten Island, New York 10305, USA

$\mathrm{Tel}+\mid 646919$ |473

Email dikshya_sh896@yahoo.com
Abstract: Mucormycosis is a rare infection caused by the ubiquitous filamentous fungi of the order Mucorales and class Zygomycetes. These species are vasotropic, causing rapid onset of tissue infarctions and necrosis and subsequent thrombosis by invading vascular bed. The disease spectrum ranges from involvement of skin, sinuses, lung, and brain to disseminated and mostly fatal infections, especially in immunocompromised hosts. Here, we present a case of a fatal disseminated mucormycosis in a 56-year-old female who had deceased donor renal allograft transplantation $\sim 2$ weeks prior to presentation. She presented with shortness of breath and dry cough. Despite being on broad-spectrum antibiotics/antifungals and proper management by transplant, infectious disease, and primary team, she died within 3 weeks of admission. Autopsy showed disseminated mucormycosis of lungs and thyroid. Disseminated infection within 2 weeks of solid organ transplantation in this patient was one of the rare features of mucormycosis.

Keywords: Zygomycetes, immunocompromised, transplant

\section{Case}

A 56-year-old black woman from South Carolina presented with complaints of shortness of breath and dry cough for 7 days. She had past medical history of end-stage renal disease status post deceased donor renal transplant nearly 2 weeks ago, dietcontrolled diabetes mellitus, hypertension, hypothyroidism, and anemia of chronic disease. Institutional approval by Northshore- LIJ Health and written informed consent from patient was obtained.

She was on maintenance immunosuppressant and prophylactic antibiotics (tacrolimus $1 \mathrm{mg}$ PO Q12h, mycophenolate $500 \mathrm{mg}$ PO Q12h, prednisone $20 \mathrm{mg}$ PO Q24h, valganciclovir $450 \mathrm{mg}$ every other day, and TMP-SMX 1/2 tab Q24h).

On initial physical examination, patient was pale looking but afebrile and on no acute distress. Her vitals on admission were blood pressure 124/74 $\mathrm{mmHg}$, pulse rate $85 / \mathrm{min}$, respiratory rate $22 / \mathrm{min}$, and saturation of $96 \%$ on $2 \mathrm{~L} / \mathrm{min}$ of oxygen via nasal cannula. Her admission labs were significant for white blood cell (WBC) count of $14,000 / \mathrm{mm}^{3}$ with neutrophilic pleocytosis, hemoglobin $7.7 \mathrm{~g} / \mathrm{dL}$, and platelet count of $1,55,000 / \mathrm{mm}^{3}$. She had normal electrolytes and liver enzymes. She was found to have acute or chronic kidney injury with serum creatinine of $2.6 \mathrm{mg} / \mathrm{dL}$ (baseline creatinine $1.5 \mathrm{mg} / \mathrm{dL}$ upon discharge 2 weeks ago). Serum levels of her immunosuppressive drugs were within normal limits. Chest X-ray on admission showed left upper lobe round infiltrate (Figure 1). The patient was treated with oxygen and multiple antimicrobials (vancomycin, aztreonam, and azithromycin) for several days. 


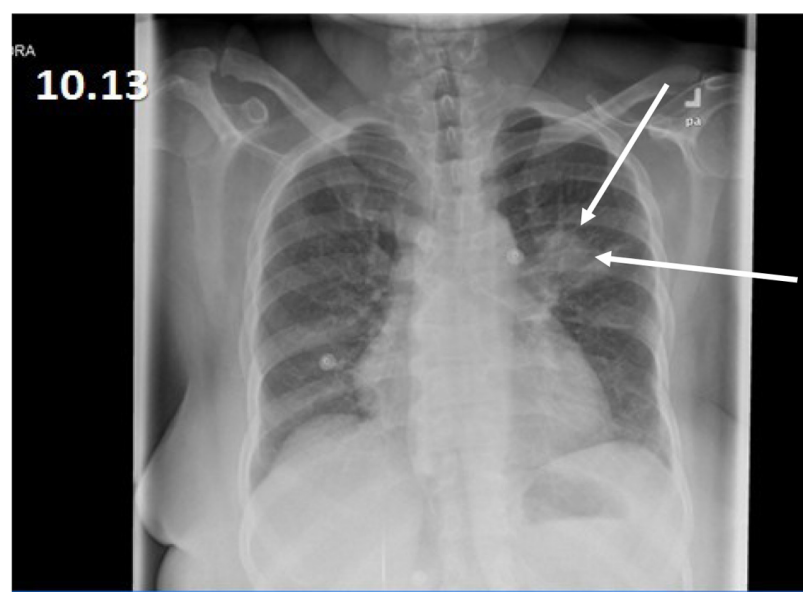

Figure I Left upper lobe round infiltrate in chest X-ray. Note: The arrow indicate the cavitary lesion in the lung.

All initial cultures including blood and sputum were negative for any bacteria or fungi. On day 5 of antimicrobial therapy, her WBC count decreased to $9,000 / \mathrm{mm}^{3}$. However, she persistently felt short of breath and continued having cough. A repeat chest X-ray showed expanding left upper lobe lesion, while computed tomography scan of chest (Figure 2) done at that time showed a left upper lobe lung lesion with cavitations, along with diffuse bilateral ground glass opacification. Due to continued cough and new left-sided chest pain which she had developed, she went for bronchoscopy with bronchoalveolar lavage and transbronchial biopsy (TBBx) nearly 10 days after admission which revealed septal fibrosis with mild chronic inflammation and focal hemosiderin-laden macrophages.

Primary transplant team added IV micafungin on her empiric antibiotic regimen after the TBBx because of no significant clinical improvement. Her doses of

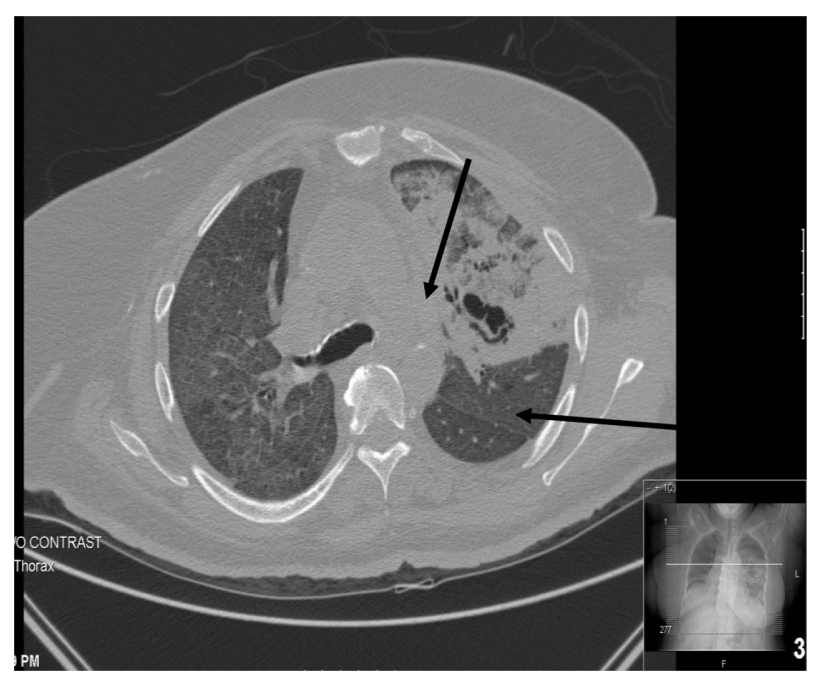

Figure 2 Computed tomography scan of chest showing left upper lobe lung lesion with cavitations, along with diffuse bilateral ground glass opacification.

Notes: The arrow indicate the cavitary lesion in the lung. The inset shows that the cavitary lesion in lung is on that level on coronal view. immunosuppressive medications were decreased as well. Samples from bronchoalveolar lavage and TBBx were negative on culture and special stains for any bacterial, mycobacterial, or fungal pathogens.

Post bronchoscopy, she started spiking temperature $\left(101.4^{\circ} \mathrm{F}\right)$ and clinically looked more lethargic and confused. Her WBC count increased to $23,000 / \mathrm{mm}^{3}$ ( $88 \%$ neutrophils). The infectious disease team was consulted nearly 2 weeks after initial admission.

The patient underwent thoracotomy with a wedge biopsy at that time. She was started on empirical IV voriconazole. Wedge biopsy results revealed diffuse alveolar hemorrhage, with hemosiderin-laden macrophages and possible capillary fibrin-platelet thrombi. However, silver stain, Gram stain, and acid-fast bacilli stain did not reveal any organisms. Again, multiple cultures for bacterial, mycobacterial, and fungal pathogens were negative. She was transferred to medical ICU after thoracotomy and remained intubated. Serum fungal markers including cryptococcal antigen, beta-D-glucan, and galactomannan antigen were negative.

Despite empiric broad-spectrum antibiotics and antifungals, she remained febrile with persistent leukocytosis, deteriorating renal function, and no improvement in oxygenation. She required five units of red blood cells transfusion post open lung biopsy for persistent anemia. Multiple attempts to wean ventilation failed. On day 20, she had massive hemoptysis followed by hypotension and bradycardia requiring cardiopulmonary resuscitation. However, she died. On autopsy, both of her lungs were heavy, boggy, and congested. There were white lines in the interlobular septa, with the right more prominent than the left, suggestive of lymphatic congestion. Left lung upper and lower lobes had a hemorrhagic appearance throughout, and blood oozed from the cut surfaces. There was a $8 \times 5 \times 2.5 \mathrm{~cm}$ wellcircumscribed hemorrhagic cavity filled with blood clot in the left upper lobe. The tracheal mucosa had bilateral ulcerative lesions $(0.5 \mathrm{~cm})$ directly beneath the vocal cords $1 \mathrm{~cm}$ from the midline. Microscopically, the fungi displayed broad-based pauci-septate hyphae with dichotomous wideangle branching in the left upper lobe hemorrhagic lesion and surrounding lung parenchyma, as well as in the pleura (Figure 3). A focus of thyroid tissue also contained these fungal hyphae (Figure 4). Associated with the fungus was necrosis and vascular thrombosis. Microbiological diagnosis of Rhizopus microsporus var. microsporus was made.

\section{Literature review}

Mucormycosis refers to a spectrum of disease presentations caused by ubiquitous saprophytic fungi of the 


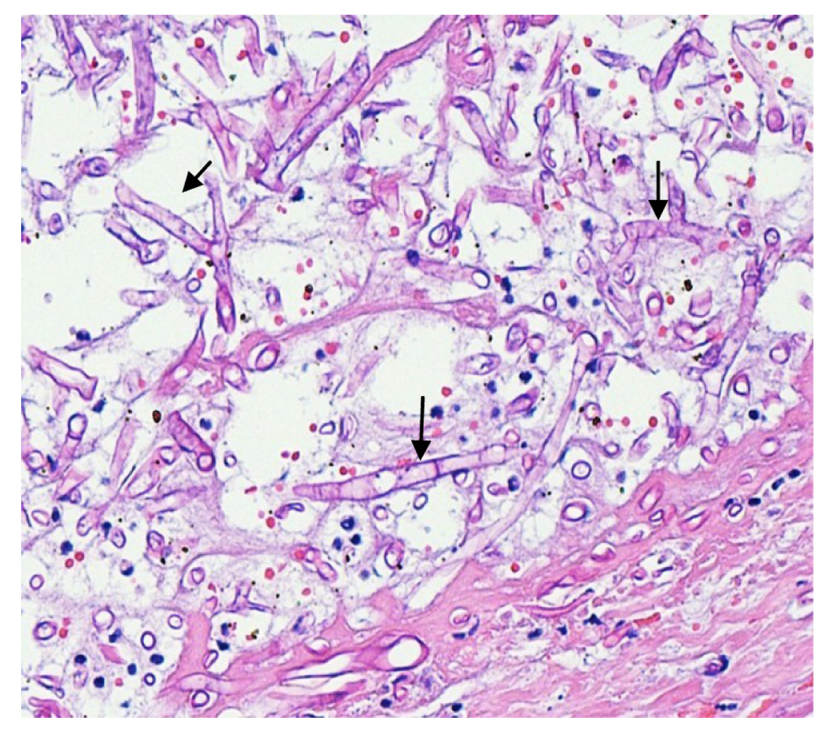

Figure 3 Broad-based pauci-septate hyphae with dichotomous wide-angle branching (arrow) on microscopic examination of Lung.

class Zygomycetes. It is a severe invasive opportunistic fungal infection that usually occurs in patients who are in immunocompromised states as HIV, diabetes, neutropenia, organ transplant, malignancy, or prolonged use of steroids. Concomitant diabetes mellitus in organ transplant recipients is the most important risk factor in developing this infection. ${ }^{1}$ Incidence of mucormycosis has been increasing over several years, due to increasing use of immunosuppressives and antifungals lacking anti-Mucorales activity such as caspofungin or voriconazole. ${ }^{2}$ Invasive fungal infections in stem cell transplant recipients account for $7.2 \%-8 \%$ and $\sim 2 \%$ in recipients of solid organ transplantation (SOT). ${ }^{3}$

It is an extremely rare condition in renal allograft recipients with an incidence of $0.2 \%-1.2 \%{ }^{4,5}$ In the multicenter TRANSNET study, it was found that the incidence of mucormycosis was $0.07 \%$ in SOT recipients in 1 year. ${ }^{6,7}$ These

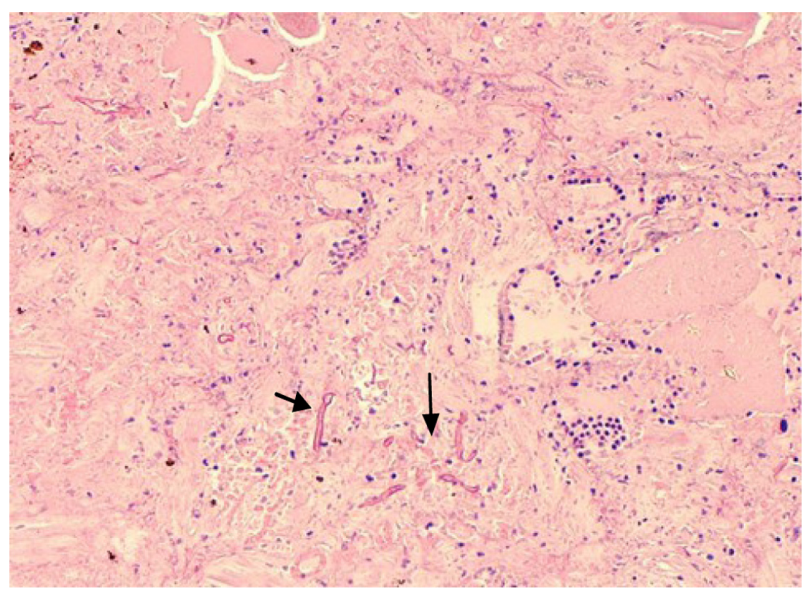

Figure 4 Broad-based pauci-septate hyphae with dichotomous wide-angle branching (arrow) on microscopic examination of thyroid. patients are more prone to develop opportunistic infections due to their immunosuppressed state. One study reported that $100 \%$ of patients received immunosuppressive, and $78.9 \%$ received a cumulative steroid dose ( $\geq 600 \mathrm{mg}$ of prednisone) therapy before diagnosis of zygomycosis. ${ }^{8}$ Our patient had several important risk factors as being an organ transplant recipient on immunosuppressive regimen, concomitant diabetes, and glucocorticoid treatment as part of her antirejection medication regimen. However, it is not clear why she had invasive fungal infection so early after transplant. Main cause of early-onset nosocomial zygomycosis can be due to donor-derived infection. ${ }^{9}$ In a recent review on 169 cases of health care-associated mucormycosis, $14 \%$ had grafttransmitted infection, representing $60 \%$ of SOT recipients. ${ }^{8}$ Environmental contamination or nosocomial source has been occasionally found to be transmitting filamentous fungi in SOT recipients. This usually occurs when there is breach in aseptic barrier while obtaining, transplanting, or implanting organ. Contamination of preservation fluid is rarely shown or suspected to be the mode of transmission of aspergillosis and mucormycosis associated with invasive fungal infections in the recipients. ${ }^{10}$ So far, 23 cases of donor-derived opportunistic filamentous fungal infections have been reported in SOT patients (71\% Aspergillus sp., 21\% Mucorales). A majority (91\%) of them were kidney transplant recipients. ${ }^{11}$ In our particular case, none of the recipient's organs from the deceased donor were reported to have invasive fungal infections or unexplained death. Preservative fluid culture was also negative for any fungal pathogens. On the other hand, non-graft-transmitted mucormycosis develops after a median of 5 months (range: 1.5-12 months) in SOT, with a significant earlier occurrence in liver transplant recipients. ${ }^{12}$ In our patient, nosocomial mucormycosis occurred after 3 weeks of transplant, which has never been reported before to best of our knowledge.

Roden et al found that the most common sites of mucormycosis in 61 SOT recipients were the lungs (37\%), skin lesions (16\%), rhinocerebral infection (16\%), sinus disease (15\%), gastrointestinal infection $(7 \%)$, disseminated disease $(2 \%)$, and other less common sites $(7 \%){ }^{13}$ Disseminated mucormycosis is defined as an involvement of two or more noncontiguous organs and usually presents with nonspecific manifestation. In a study done on ten SOT recipients with zygomycosis, $12.9 \%$ developed disseminated mucormycosis. The majority had lungs involved which was identified on autopsy or with a cavitary radiologic appearance highly suggestive of invasive fungal infection. ${ }^{1}$ In our case, involvement of lung and thyroid tissue classifies it as a disseminated infection. The case described is a unique case of 
disseminated mucormycosis because only three documented human infections owing to this organism ( $R$. microsporus var. microsporus) have been described so far. ${ }^{14-16}$ None of the published cases were associated with SOT recipients, however.

In one of the largest study done on mucormycosis in a renal transplant patient, it was found that there is no statistically significant difference in mortality rate between sex, different age, and time from transplant. Conclusion from this 2 decades of study was that mucormycosis after renal transplantation has a poor prognosis, particularly in patients with pulmonary involvement. ${ }^{17}$

The mortality rate among SOT recipients with zygomycosis has typically ranged from $49 \%$ to $71 \%$ and up to $96 \%$ in disseminated form of infection. ${ }^{18,19}$ Successful treatment requires a combination of early diagnosis, reversal of underlying risk factors, reduction of immunosuppression, prompt administration of antifungal therapy (amphotericin $\mathrm{B}$, the drug of choice) if possible, and aggressive surgical debridement when applicable. A high index of suspicion is required for rapid and timely diagnosis of invasive zygomycosis, preferably by culture and histological confirmation. Diagnosis of mucormycosis is very difficult due to the rarity of the problem and significant limitations of diagnostic tests. In our particular patient, definitive tests included fungal cultures and lung biopsy. However, both test missed the diagnosis most likely due to sampling bias or inadequate tissue. Many missed cases of these deep-seated fungal infections are diagnosed on autopsy. In fact, autopsy series have demonstrated that up to $50 \%$ of cases of mucormycosis are diagnosed postmortem. ${ }^{20}$ Another study reviewed autopsies with SOT in UCLA Medical Center (Santa Monica, CA, USA), especially focusing on the deep-seated fungal infection, and the incidence of fungal infections, causative fungi, and organs involved were evaluated. Deep-stated fungal infections were demonstrated in $21.0 \%$ of the patients with SOT. The incidence of fungal infections was $26.1 \%$ in kidney transplantation, which was the highest rate in SOT autopsies. The most common causative fungi were Aspergillus sp., seen in $70.6 \%$ of SOT autopsies. ${ }^{21}$

From our case report, we showed that invasive fungal infection can occur very early after transplant and should be regarded as differential when these patients present with nonspecific clinical feature with no identified source of infection.

\section{Disclosure}

The authors report no conflicts of interest in this work.

\section{References}

1. Almyroudis NG, Sutton DA, Linden P, et al. Zygomycosis in solid organ transplant recipients in a tertiary transplant center and review of the literature. Am J Transpl. 2006;6(10):2365-2367.

2. Vigouroux S, Morin O, Moreau P. et al. Zygomycosis after prolonged use of voriconazole in immunocompromised patients with hematologic disease: attention required. Clin Infect Dis. 2005;40(4):35-37.

3. Rammaert B, Lanternier F, Zahar JR, et al. Healthcare-associated mucormycosis. Clin Infect Dis. 2012;54(Suppl 1):S44-S54.

4. Nampoory MR, Khan ZU, Johny KV, et al. Invasive fungal infections in renal transplant recipients. $J$ Infect. 1996;33:95-101.

5. Chkhotua A, Yussim A, Tovar A, et al. Mucormycosis of the renal allograft: case report and review of the literature. Transpl Int. 2001;14(6):438-441.

6. Pappas PG, Alexander BD, Andes DR, et al. Invasive fungal infections among organ transplant recipients: results of the Transplant-Associated Infection Surveillance Network (TRANSNET). Clin Infect Dis. 2010;50:1101-1111.

7. Park BJ, Pappas PG, Wannemuehler KA, et al. Invasive non-Aspergillus mold infections in transplant recipients, United States, 2001-2006. Emerg Infect Dis. 2011;17:1855-1864.

8. Rammaert B, Lanternier F, Zahar JR, et al. Healthcare-associated mucormycosis. Clin Infect Dis. 2012;54(Suppl 1):S44-S54.

9. Lanternier F, Sun HY, Ribaud P, et al. Mucormycosis in organ and stem cell transplant recipients. Clin Infect Dis. 2012;54(11):1629-1636.

10. Singh NM, Husain S. Aspergillosis in solid organ transplantation and the AST Infectious Diseases Community of Practice. Am J Transpl. 2013;13(4):228-241.

11. Carlos F, Nina S. Donor-derived filamentous fungal infections in solid organ transplant recipients. Curr Opin Infect Dis. 2013;26(4):309-316.

12. Singh N, Aguado JM, Bonatti H, et al. Zygomycosis in solid organ transplant recipients: a prospective, international, matched case control study to assess risk factors and outcome. J Infect Dis. 2009;200:1002-1011.

13. Roden MM, Zaoutis TE, Buchanan WL, et al. Epidemiology and outcome of zygomycosis: a review of 929 reported cases. Clin Infect Dis. 2005;41:634-653.

14. Kerr PG, Turner, H, Davidson A, Bennett C, Maslen M. Zygomycosis requiring amputation of the hand: an isolated case in a patient receiving hemodialysis. Med J Aust. 1988;148:258-259.

15. Kwon-Chung KJ, Bennett JE. Medical Mycology. Lea \& Febiger, Philadelphia, PA, USA and London, UK. 1992.

16. West BC, Oberle AD, Kwon-Chumg KJ. Mucormycosis caused by Rhizopus microsporus var. microsporus: cellulitis in the leg of a diabetic patient cured by amputation. J Clin Microbiol. 1995;33;3341-3344.

17. Einollahi B, Lessan-Pezeshki M, Aslani J, et al. Two decades of experience in Mucormycosis after kidney transplantation. Ann Transpl. 2011;16(3):44-48.

18. Sun HY, Singh N. Mucormycosis: its contemporary face and management strategies. Lancet Infect Dis. 2011;11(4):301-311.

19. Aslani J, Eizadi M, Kardavani B, et al. Mucormycosis after kidney transplantations: report of seven cases. Scand J Infect Dis. 2007; 39(8):703-706.

20. Kauffman CA, Pappas PG, Sobel JD, Dismukes WE, editors. Essentials of Clinical Mycology. 2nd ed. New York, NY, USA, Springer; 2011.

21. Wakayama M, Shibuya K, Ando T, et al. Deep-seated mycosis in patients with solid-organ transplantation - a study of autopsied cases in the United States. J Jpn Assoc Infect Dis. 2000;74(4):378-386. 
International Medical Case Reports Journal

Dovepress

\section{Publish your work in this journal}

The International Medical Case Reports Journal is an international, peer-reviewed open-access journal publishing original case reports from all medical specialties. Previously unpublished medical posters are also accepted relating to any area of clinical or preclinical science. Submissions should not normally exceed 2,000 words or

4 published pages including figures, diagrams and references. The manuscript management system is completely online and includes a very quick and fair peer-review system, which is all easy to use. Visit $\mathrm{http}: / / \mathrm{www}$.dovepress.com/testimonials.php to read real quotes from published authors.

Submit your manuscript here: http://www.dovepress.com/international-medical-case-reports-journal-journal 